\title{
Isoperimetric inequalities for soap-film-like surfaces spanning nonclosed curves
}

\section{KeOmKYo Seo}

For a soap-film-like surface $\Sigma$ spanning a nonclosed curve $\Gamma$ in $\mathbf{R}^{n}$, it is proved that

$$
4 \pi \operatorname{Area}(\Sigma) \leq \operatorname{Length}(\Gamma)^{2}
$$

In the upper hemisphere $\mathbf{S}_{+}^{n}$ we use a mixed area $M_{p}(\Sigma)$, which was introduced by Choe and Gulliver [9] to prove an isoperimetric inequality

$$
4 \pi M_{p}(\Sigma) \leq \operatorname{Length}(\Gamma)^{2}
$$

for a soap-film-like surface $\Sigma$ with nonclosed boundary $\partial \Sigma$. In a complete simply connected Riemannian manifold with sectional curvature bounded above by a nonpositive constant $K$, a soapfilm-like surface $\Sigma$ with an embedded, connected, and nonclosed boundary curve $\Gamma$ satisfies

$$
4 \pi \operatorname{Area}(\Sigma)-K \operatorname{Area}(\Sigma)^{2} \leq \operatorname{Length}(\Gamma)^{2} .
$$

Moreover, for a soap-film-like surface $\Sigma$ with nonclosed boundary $\partial \Sigma$ in a complete simply connected Riemannian manifold $M$ with sectional curvature bounded above by a constant $K$, we obtain a weak isoperimetric inequality

$$
2 \pi \operatorname{Area}(\Sigma)-K \operatorname{Area}(\Sigma)^{2} \leq \operatorname{Length}(\partial \Sigma)^{2} .
$$

Finally, we prove that a soap-film-like surface $\Sigma \subset \mathbf{R}^{n}$ satisfies

$$
2 \sqrt{2} \pi \operatorname{Area}(\Sigma) \leq \operatorname{Length}(\partial \Sigma)^{2} .
$$

\section{Introduction}

It has been a long-standing conjecture that the classical isoperimetric inequality

$$
4 \pi \operatorname{Area}(\Sigma) \leq \operatorname{Length}(\partial \Sigma)^{2}
$$


should hold for any compact smooth minimal surface $\Sigma$ in $\mathbf{R}^{n}$. In 1921, it was partially proved by Carleman [5] for simply connected minimal surfaces in $\mathbf{R}^{n}$. Osserman and Schiffer [16] showed that the isoperimetric inequality (1.1) still holds with a strict inequality for doubly connected minimal surfaces in $\mathbf{R}^{3}$, using the Weierstrass representation formula. Later Feinberg [12] generalized this to doubly connected minimal surfaces in $\mathbf{R}^{n}$ for all $n$. In 1984, Li et al. [15] proved the inequality (1.1) for minimal surfaces in $\mathbf{R}^{3}$ with one or two boundary components, introducing the concept of weakly connected boundary. Choe [6] later showed that the inequality (1.1) holds also for any minimal surfaces in $\mathbf{R}^{n}$ with one or two boundary components. However, this conjecture is still open for minimal surfaces with at least three boundary components.

On the other hand, one might propose the same conjecture for soap-filmlike surfaces with singularities. Almgren [1] showed that the conjecture is true for area minimizing flat chains mod $k$, using geometric measure theory. In [7], Choe proved that the inequality (1.1) holds also for two-dimensional stationary varifolds with connected boundary of multiplicity $\geq 1$. However, until now, it was unknown whether soap-film-like surfaces with nonclosed boundary satisfy this isoperimetric inequality. Physically such surfaces may be observed in soap-film experiments, where the nonclosed boundary curve is connected by a singular curve in the interior of surface and three surfaces meet along the singular curve. The mathematical existence of such soapfilm-like surfaces was studied by Parks [17] and Drachman and White [10]. In this paper, we study isoperimetric inequalities for soap-film-like surfaces whose boundary contains a nonclosed curve.

It turns out that for a soap-film-like surface $\Sigma$ spanning a nonclosed curve $\Gamma$ in $\mathbf{R}^{n}$ (Theorem 3.4),

$$
4 \pi \operatorname{Area}(\Sigma) \leq \operatorname{Length}(\Gamma)^{2} .
$$

We emphasize that the above inequality does not contain the length of singular curves of $\Sigma$. The key idea of the proof of this inequality is the following: firstly, we obtain the area and density comparison between $\Sigma$ and $p \circledast \Gamma$ for a point $p \in \Sigma$. Secondly, we prove the isoperimetric inequality for the cone $p \circledast \Gamma$, where $p \circledast \Gamma$ is the union of the line segments from $p$ to $q$ over all $q \in \Gamma$. Note that we cannot apply Stokes' theorem to $\Sigma$ because $\Sigma$ is singular, hence it is nonorientable in general. For that reason, we use a classical minimal surface $\widetilde{\Sigma}$ which is obtained by cutting the soap-film-like surface $\Sigma$ along the singular curves, hence the boundary of $\widetilde{\Sigma}$ is the union of the boundary 
curve $\Gamma$ of $\Sigma$ and the singular curves. Thereafter, with these inequalities, we finally obtain the desired isoperimetric inequality for soap-film-like surfaces.

We are also concerned with isoperimetric inequalities for soap-film-like surfaces in a curved space. Given a point $p \in \mathbf{S}^{n}$, using a modified area $M_{p}(\Sigma)$ which was introduced by Choe and Gulliver [9], we shall obtain the following isoperimetric type inequality for a soap-film-like surface $\Sigma$ in the upper hemisphere (Theorem 4.6)

$$
4 \pi M_{p}(\Sigma) \leq \operatorname{Length}(\partial \Sigma)^{2}
$$

Moreover, we obtain an isoperimetric inequality for a soap-film-like surface $\Sigma$ in a complete simply connected Riemannian manifold $M$ with sectional curvature bounded above by a nonpositive constant $K$ (Theorem 5.2):

$$
4 \pi \operatorname{Area}(\Sigma)-K \text { Area }(\Sigma)^{2} \leq \operatorname{Length}(\partial \Sigma)^{2}
$$

Finally, without topological assumption on boundary it is verified that a weaker isoperimetric inequality

$$
2 \pi \operatorname{Area}(\Sigma)-K \operatorname{Area}(\Sigma)^{2} \leq \operatorname{Length}(\partial \Sigma)^{2}
$$

holds for a soap-film-like surface $\Sigma$ in $M$ (Theorem 6.1). In particular, when $M=\mathbf{R}^{n}$, the inequality (1.3) can be improved as follows (Theorem 6.2 ):

$$
2 \sqrt{2} \pi \operatorname{Area}(\Sigma) \leq \operatorname{Length}(\partial \Sigma)^{2}
$$

\section{Preliminaries}

Let $\Gamma$ be an embedded (possibly nonclosed) piecewise $C^{1}$-curve in a Riemannian manifold $M$. We will consider singular surfaces which are orientable $C^{2}$-surfaces except the curve $\Gamma$ and the singular curves $\cup C_{i}$, such that each $C_{i}$ is a piecewise $C^{1}$-curve and $C^{1}$ up to $\Gamma \cup\left(\cup C_{i}\right)$.

Definition 2.1 [11]. A rectifiable varifold $\Sigma$ in $M$ is called strongly stationary with respect to $\Gamma$ if for every smooth variation $\phi: \mathbf{R} \times M \rightarrow M$ with $\phi(0, x) \equiv x$, we have

$$
\left.\frac{\mathrm{d}}{\mathrm{d} t}\right|_{t=0}(\operatorname{Area}(\phi(t, \Sigma))+\operatorname{Area}(\phi([0, t] \times \Gamma))) \geq 0 .
$$



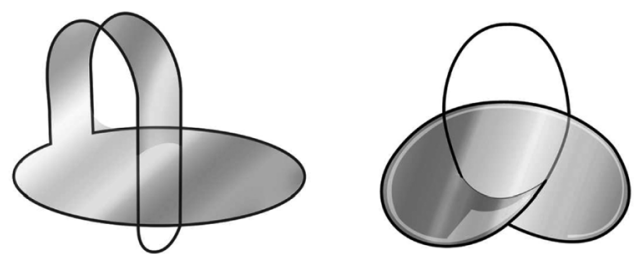

Figure 1: Soap-film-like surfaces with nonclosed boundary.

Recall that a compactly supported rectifiable varifold $\Sigma$ is said to be stationary in $M \backslash \Gamma$ if

$$
\left.\frac{\mathrm{d}}{\mathrm{d} t}\right|_{t=0} \operatorname{Area}(\phi(t, \Sigma))=0
$$

for all smooth variation $\phi: \mathbf{R} \times M \rightarrow M$ such that $\phi(0, x) \equiv x$ and $\phi(t, \xi) \equiv$ $\xi$ for $\xi$ in a neighborhood of $\Gamma$. It follows that the strong stationarity of $\Sigma$ implies that $\Sigma$ is stationary in $M \backslash \Gamma$.

If we choose the variational vector field $X=\phi^{\prime}(0, x)$ to preserve the singular curves $\cup C_{i}$, then the stationarity condition implies that $H \equiv 0$ on $\Sigma \backslash \cup C_{i}$, that is, $\Sigma \backslash \cup C_{i}$ is minimal. Furthermore, if $\Sigma$ is stationary in $M \backslash \Gamma$, then the first variation formula of the area [18] implies

$$
\int_{\cup C_{i}} \sum_{j \in J(p)}\left\langle\nu_{j}(p), X^{\perp}(p)\right\rangle d s(p)=0,
$$

where $X^{\perp}$ is the normal component of the variational vector field $X=$ $\phi^{\prime}(0, x), J(p)$ indexes the collection of surfaces $\Sigma_{j}$ 's which meet at a point $p$ in $\cup C_{i} \backslash \Gamma$ and $\nu_{j}$ is the outward unit conormal vector to $\partial \Sigma_{j}$ along $\cup C_{i}$, where the surfaces $\Sigma_{j}$ 's meet. Since the choice of the variational vector field $X$ is arbitrary along $\cup C_{i} \backslash \Gamma$, it follows that

$$
\sum_{j} \nu_{j}(p)=0 \text { on } \cup C_{i} \backslash \Gamma,
$$

which is called the balancing of $\nu_{j}$ along the singular curves of $\Sigma$ [14]. For an area minimizing surface $\Sigma$, it is well known that along the singular curves three surfaces meet at $120^{\circ}([2,20])$, hence from $(2.1)$ it follows that $\nu_{1}(p)+$ $\nu_{2}(p)+\nu_{3}(p)=0$ for $p \in \cup C_{i} \backslash \Gamma$.

Unfortunately there is no obvious definition of boundary of $\Sigma$, since $\Sigma$ is not orientable in general. Hence, we define the boundary of $\Sigma$ in variational terms as follows. 
Definition 2.2 $[11, \mathbf{1 4}] . \quad \Gamma$ is said to be variational boundary of a surface $\Sigma$ if there exists an $\mathcal{H}^{1}$ measurable vector field $\nu_{\Sigma}$ along $\Gamma$ which is orthogonal to $\Gamma$, with $\left|\nu_{\Sigma}\right| \leq 1$, such that for every smooth vector field $X$ defined on $M$,

$$
\int_{\Sigma} \operatorname{div} X^{\mathrm{T}} \mathrm{d} A=\int_{\Gamma}\left\langle X, \nu_{\Sigma}\right\rangle \mathrm{d} s .
$$

We denote by $\mathcal{S}$ the class of singular surfaces $\{\Sigma\}$ that satisfy the following:

- $\Sigma$ is orientable and $C^{2}$ except the piecewise $C^{1}$ variational boundary $\partial \Sigma$ and the singular curves $\cup C_{i}$, where each $i$ is a piecewise $C^{1}$ curve.

- $\Sigma$ is $C^{1}$ up to $\partial \Sigma \cup\left(\cup C_{i}\right)$.

Given a strongly stationary surface $\Sigma$ in $\mathcal{S}$ spanning a nonclosed curve $\Gamma$, one can see that the variational boundary $\partial \Sigma$ of $\Sigma$ is exactly equal to $\Gamma$. Therefore we will call $\Gamma$ the boundary $\partial \Sigma$ of $\Sigma$ for short as in a classical surface theory. In fact, strong stationarity for surfaces in the class $\mathcal{S}$ is equivalent to stationarity in $M \backslash \Gamma$ plus the above boundary condition. Throughout this paper we study an isoperimetric inequality for strongly stationary surfaces in $\mathcal{S}$.

Recall that the density $\Theta(\Sigma, p)$ of a surface $\Sigma$ at a point $p$ in a Riemannian manifold $M$ is defined to be

$$
\Theta(\Sigma, p)=\lim _{\varepsilon \rightarrow 0} \frac{\operatorname{Area}\left(\Sigma \cap B_{\varepsilon}(p)\right)}{\pi \varepsilon^{2}},
$$

where $B_{\varepsilon}(p)$ is the geodesic ball of $M$ centered at $p$ with radius $\varepsilon$. Note that for $\Sigma$ is a surface in $\mathcal{S}$, the density $\Theta(\Sigma, p)$ is well-defined and we may also compute the density in terms of length:

$$
\Theta(\Sigma, p)=\lim _{\varepsilon \rightarrow 0} \frac{\text { Length }\left(\Sigma \cap \partial B_{\varepsilon}(p)\right)}{2 \pi \varepsilon} .
$$

We also define the two-dimensional angle $\operatorname{Angle}(\Sigma, p)$ of $\Sigma$ from $p$ as follows:

$$
\operatorname{Angle}(\Sigma, p)=2 \pi \Theta(\Sigma, p)
$$

\section{Isoperimetric inequalities for soap-film-like surfaces in Euclidean space}

Let $\Gamma$ be a Jordan curve in $\mathbf{R}^{n}$ and $\Sigma$ be a minimal surface in $\mathbf{R}^{n}$ with boundary $\Gamma$. Then for a point $p \in \Sigma \backslash \Gamma$, it has been known that the density 
comparison between $\Sigma$ and the cone $p \circledast \Gamma$ over $p$ satisfies

$$
\Theta(\Sigma, p) \leq \Theta(p \circledast \Gamma, p),
$$

where equality holds only for $\Sigma=p * \Gamma$. (See $[6,11,13]$.) Recently Gulliver and Yamada [14] proved that the density comparison (3.1) still holds for a strongly stationary surface $\Sigma$ spanning a graph $\Gamma$. For a soap-film-like surface $\Sigma$ in $\mathbf{R}^{n}$ with nonclosed boundary $\Gamma$, the density comparison (3.1) is still true. More precisely, we have the following.

Proposition 3.1. Let $\Sigma \in \mathcal{S}$ be a strongly stationary surface in $\mathbf{R}^{n}$ with an embedded, connected, and nonclosed boundary curve $\Gamma$. Then for a point $p \in \Sigma \backslash \Gamma$ we have

$$
\Theta(\Sigma, p)<\Theta(p \circledast \Gamma, p),
$$

unless $\Sigma$ itself is a cone $p \times \Gamma$.

Remark. It follows from this proposition that $1 \leq \Theta(p \circledast \Gamma, p)$ for $p \in \Sigma \backslash \Gamma$ and in particular $\frac{3}{2} \leq \Theta(p \circledast \Gamma, p)$ for a point $p$ on the singular curves of $\Sigma \backslash \Gamma$. The proof of this proposition is similar to that of Theorem 1 in [14].

Proof. Let $\cup C_{i}$ be the set of singular curves of $\Sigma$ satisfying that each $C_{i}$ is a piecewise $C^{1}$-curve as mentioned in previous section. We cut the surface $\Sigma$ along the singular curves $\cup C_{i}$ to obtain a $C^{2}$-surface $\widetilde{\Sigma}$, such that $\widetilde{\Sigma}$ is a minimal surface in $\mathbf{R}^{n}$ with $\partial \widetilde{\Sigma}=\Gamma \cup\left(\cup C_{i}\right)$.

For the Euclidean distance function $r(x)=\operatorname{dist}(p, x)$, from [6] we see that

$$
2 \pi \delta_{p} \leq \Delta \log r .
$$

Therefore

$$
\begin{aligned}
2 \pi \Theta(\Sigma, p)=2 \pi \Theta(\widetilde{\Sigma}, p) & \leq \int_{\widetilde{\Sigma}} \Delta \log r \\
& =\int_{\partial \widetilde{\Sigma}} \frac{1}{r} \frac{\partial r}{\partial \nu} \\
& =\int_{\Gamma} \frac{1}{r} \frac{\partial r}{\partial \nu}+\int_{\cup C_{i}} \frac{1}{r} \frac{\partial r}{\partial \nu_{j}} \\
& =\int_{\Gamma} \frac{1}{r} \frac{\partial r}{\partial \nu},
\end{aligned}
$$


where $\nu$ is the outward unit conormal vector to $\Gamma$ at a point $q \in \Gamma \backslash \cup C_{i}$ and $\nu_{j}$ is the outward unit conormal vector to $\partial \Sigma_{j}$ along $\cup C_{i}$, where the surfaces $\Sigma_{j}$ 's meet

Similarly, we may compute the density of the cone $p \circledast \Gamma$ at $p \in \Sigma \backslash \Gamma$. Since $2 \pi \delta_{p}=\Delta \log r$ on $p \nVdash \partial \Sigma$, it follows that

$$
\begin{aligned}
2 \pi \Theta(p \circledast \Gamma, p) & =\int_{p \circledast \Gamma} \Delta \log r \\
& =\int_{\Gamma} \frac{1}{r} \frac{\partial r}{\partial \eta}+\int_{\overline{p a}} \frac{1}{r} \frac{\partial r}{\partial \eta}+\int_{\overline{p b}} \frac{1}{r} \frac{\partial r}{\partial \eta}=\int_{\Gamma} \frac{1}{r} \frac{\partial r}{\partial \eta}
\end{aligned}
$$

where the points $a$ and $b$ are the 4 s of $\Gamma$ and $\eta$ is the outward unit conormal to $p \circledast \Gamma$. In the last equality above, we used the fact that $\frac{\partial r}{\partial \eta}$ vanishes along $\overline{p a}$ and $\overline{p b}$ because $\nabla r$ and $\eta$ are perpendicular. On the other hand, it is easy to see that

$$
\frac{\partial r}{\partial \nu} \leq \frac{\partial r}{\partial \eta} \leq 1 \text { on } \Gamma
$$

Hence from the inequalities (3.3) and (3.4), we get

$$
\begin{aligned}
2 \pi \Theta(\Sigma, p)=2 \pi \Theta(\widetilde{\Sigma}, p) & \leq \int_{\Gamma} \frac{1}{r} \frac{\partial r}{\partial \nu} \\
& \leq \int_{\Gamma} \frac{1}{r} \frac{\partial r}{\partial \eta} \quad(\text { by }(3.5)) \\
& =\int_{p \circledast \Gamma} \Delta \log r=2 \pi \Theta(p æ \Gamma, p) .
\end{aligned}
$$

Proposition 3.2. Let $\Sigma \in \mathcal{S}$ be a strongly stationary surface in $\mathbf{R}^{n}$ with an embedded, connected, and nonclosed boundary curve $\Gamma$. Then for a point $p \in \mathbf{R}^{n}$ we have

$$
\operatorname{Area}(\Sigma) \leq \operatorname{Area}(p æ \Gamma)
$$

Proof. As in the proof of Proposition 3.1, we cut the surface $\Sigma$ along the singular curves $\cup C_{i}$ to obtain a minimal surface $\widetilde{\Sigma}$ with $\partial \widetilde{\Sigma}=\Gamma \cup\left(\cup C_{i}\right)$. Let 


$$
\begin{aligned}
& r(x)=\operatorname{dist}(p, x), \text { for } x \in \widetilde{\Sigma} \text {. Using } \Delta r^{2}=4 \text { on } \widetilde{\Sigma}, \text { we get } \\
& 4 \operatorname{Area}(\Sigma)=4 \operatorname{Area}(\widetilde{\Sigma})=\int_{\partial \widetilde{\Sigma}} 2 r \frac{\partial r}{\partial \nu}=\int_{\Gamma} 2 r \frac{\partial r}{\partial \nu}+\int_{\cup C_{i}} 2 r \frac{\partial r}{\partial \nu_{j}}=\int_{\Gamma} 2 r \frac{\partial r}{\partial \nu},
\end{aligned}
$$

where $\nu$ is the outward unit conormal vector to $\Gamma$ at a point $q \in \partial \Sigma \backslash \cup C_{i}$ and $\nu_{j}$ is the outward unit conormal vector to $\partial \Sigma_{j}$ along $\cup C_{i}$, where the surfaces $\Sigma_{j}$ 's meet. Furthermore on the cone $p \circledast \partial \Sigma$, the identity $\Delta r^{2}=4$ still holds. Hence

$$
4 \operatorname{Area}(p \circledast \Gamma)=\int_{\Gamma} 2 r \frac{\partial r}{\partial \eta}+\int_{\overline{p a}} 2 r \frac{\partial r}{\partial \eta}+\int_{\overline{p b}} 2 r \frac{\partial r}{\partial \eta}=\int_{\Gamma} 2 r \frac{\partial r}{\partial \eta},
$$

where the points $a$ and $b$ are the endpoints of $\Gamma$ and $\eta$ is the outward unit conormal to $p \circledast \Gamma$ and we used the fact that $\frac{\partial r}{\partial \eta}=\langle\nabla r, \eta\rangle=0$ on $\overline{p a}$ and $\overline{p b}$ in the last inequality. It follows from the inequality (3.5) that

$$
4 \operatorname{Area}(\Sigma) \leq \int_{\Gamma} 2 r \frac{\partial r}{\partial \nu} \leq \int_{\Gamma} 2 r \frac{\partial r}{\partial \eta}=4 \operatorname{Area}(p * \Gamma) .
$$

In order to prove an isoperimetric inequality for soap-film-like surfaces, we need the following isoperimetric inequality for cones.

Lemma 3.3 [6]. Let $C(t)$ be a connected plane curve parameterized by the angle from the origin 0 , for $a \leq t \leq b$. If $\operatorname{dist}(0, C)>0, b-a \geq 2 \pi$, and $\operatorname{dist}(0, C(a))=\operatorname{dist}(0, C(b))$, then

$$
4 \pi \text { Area }(0 ※ C) \leq \operatorname{Length}(C)^{2} .
$$

Equality holds if and only if $C$ is a circle and $b-a=2 \pi$, where Area $(0 \circledast C)$ counts multiplicity, and $C(t)$ may be a line segment which is a subset of a ray emanating from the origin for some values of $t$.

Theorem 3.4. Let $\Sigma \in \mathcal{S}$ be a strongly stationary surface in $\mathbf{R}^{n}$ with an embedded, connected, and nonclosed boundary curve $\Gamma$. Then we have

$$
4 \pi \operatorname{Area}(\Sigma) \leq \operatorname{Length}(\Gamma)^{2} .
$$

Proof. For the endpoint $a$ and $b$ of $\Gamma$, there exists a hyperplane $\Pi \in \mathbf{R}^{n}$ which bisects and is perpendicular to the line segment $\overline{a b}$. For any $p \in(\Sigma \cap \Pi) \backslash \Gamma$, 
we have

$$
\operatorname{dist}(p, a)=\operatorname{dist}(p, b) \text { and } 1 \leq \Theta(p \circledast \Gamma, p)=\frac{1}{2 \pi} \operatorname{Angle}(\Gamma, p) .
$$

Since $\Gamma$ is connected, we can develop the cone $p \circledast \Gamma$ into a cone $O \circledast C$ on $\mathbf{R}^{2}$, such that $C$ has the same length as $\Gamma$. Here, Area $(p \notin \Gamma)$ counts multiplicity. Then, by applying Lemma 3.3, we can obtain

$$
4 \pi \operatorname{Area}(p \circledast \Gamma) \leq \operatorname{Length}(\Gamma)^{2} .
$$

Hence the conclusion follows from Proposition 3.2.

We say that a set $C \subset \mathbf{R}^{n}$ is radially connected from a point $p \in \mathbf{R}^{n}$ if $\{r: r=\operatorname{dist}(p, q), q \in C\}$ is a connected interval. Obviously, every connected set in $\mathbf{R}^{n}$ is radially connected from any point $p \in \mathbf{R}^{n}$. In general, it turns out that Theorem 3.4 holds for a soap-film-like surface whose boundary is radially connected and the number of the boundary components is at least 2. More precisely,

Theorem 3.5. Let $\Sigma \in \mathcal{S}$ be a strongly stationary surface in $\mathbf{R}^{n}$ with boundary $\partial \Sigma$. Assume that at least one component of $\partial \Sigma$ is a nonclosed curve with endpoints $a$ and $b$. Assume also that there exists a point $p \in \Sigma \backslash \partial \Sigma$, such that $\operatorname{dist}(p, a)=\operatorname{dist}(p, b)$ and $\partial \Sigma$ is radially connected from $p$. Then we have

$$
4 \pi \operatorname{Area}(\Sigma) \leq \operatorname{Length}(\partial \Sigma)^{2}
$$

Proof. The proof is based on that of Theorem 3.4. Let $\Gamma_{i}(1 \leq i \leq m)$ be the (possibly empty) set of the closed curves among the boundary $\partial \Sigma$ and let $\Gamma_{0}$ be the nonclosed curve with endpoints $a$ and $b$ from our assumption. We can develop a space curve $C$ onto a plane curve $\bar{C}$ in such a way that under a suitable parameterization of $\bar{C}, \bar{C}$ winds around $p$ counterclockwise at all points of $\bar{C}$ except for a (possibly empty) subset which is a subset of rays emanating from $p$. For each closed curve $\Gamma_{i}(1 \leq i \leq m)$, choose a point $q_{i} \in \Gamma_{i}$, such that $\operatorname{dist}\left(p, q_{i}\right)=\operatorname{dist}\left(p, \Gamma_{i}\right)$ and develop the nonclosed curve $\Gamma_{i} \backslash\left\{q_{i}\right\}$ onto a plane curve $\bar{\Gamma}_{i}$ in $\mathbf{R}^{2}$ as above. Similarly, when $i=0$, we develop the nonclosed curve $\bar{\Gamma}_{0}$ in $\mathbf{R}^{2}$.

Let $q_{i}^{1}$ and $q_{i}^{2}$ be the two endpoints of $\bar{\Gamma}_{i}$ for $0 \leq i \leq m$. In particular, $q_{0}^{1}=a$ and $q_{0}^{2}=b$. Note that $\operatorname{dist}\left(p, \bar{\Gamma}_{i}>0\right.$ for all $i$ by assumption that 
$p \in \Sigma \backslash \partial \Sigma$. Moreover, we have

$$
\operatorname{dist}\left(p, q_{i}^{1}\right)=\operatorname{dist}\left(p, q_{i}^{2}\right)
$$

for $0 \leq i \leq m$. Without loss of generality, we may rearrange $\bar{\Gamma}_{i}$ 's and assume that

$$
\operatorname{dist}\left(p, q_{0}^{1}\right) \leq \operatorname{dist}\left(p, q_{1}^{1}\right) \leq \cdots \leq \operatorname{dist}\left(p, q_{m}^{1}\right) .
$$

Since the boundary $\partial \Sigma$ is radially connected from $p$, we see that for every $0 \leq i \leq m$, there exists a point $r_{i}$ and $k=k(i) \in\{0,1, \ldots, i-1\}$ satisfying that

$$
r_{i} \in \bar{\Gamma}_{k} \quad \text { and } \quad \operatorname{dist}\left(p, r_{i}\right)=\operatorname{dist}\left(p, q_{i}^{1}\right)
$$

Thus we choose $r_{1} \in \bar{\Gamma}_{0}$, such that $\operatorname{dist}\left(p, r_{1}\right)=\operatorname{dist}\left(p, q_{1}^{1}\right)$. Then we construct a plane curve $\widetilde{\Gamma}_{1}$ by cutting $\bar{\Gamma}_{0}$ at the point $r_{1} \in \bar{\Gamma}_{0}$ and inserting $\bar{\Gamma}_{1}$ into $\bar{\Gamma}_{0}$. By this process, $\widetilde{\Gamma}_{1}$ is a connected plane curve and its endpoints $\tilde{q}_{1}^{1}$ and $\tilde{q}_{1}^{2}$ satisfy

$$
\operatorname{dist}\left(p, \tilde{q}_{1}^{1}\right)=\operatorname{dist}\left(p, \tilde{q}_{1}^{2}\right) .
$$

Again by radial connectedness of $\partial \Sigma$, there exists a point $r_{2} \in \widetilde{\Gamma}_{1}$, such that $\operatorname{dist}\left(p, r_{2}\right)=\operatorname{dist}\left(p, q_{1}^{2}\right)$. As above, we construct a curve $\widetilde{\Gamma}_{2}$ by cutting $\widetilde{\Gamma}_{1}$ at the point $r_{2} \in \widetilde{\Gamma}_{1}$ and inserting $\bar{\Gamma}_{2}$ into $\widetilde{\Gamma}_{1}$. Here, the endpoints of $\widetilde{\Gamma}_{2}$, say $\tilde{q}_{2}^{1}$ and $\tilde{q}_{2}^{2}$ satisfy

$$
\operatorname{dist}\left(p, \tilde{q}_{2}^{1}\right)=\operatorname{dist}\left(p, \tilde{q}_{2}^{2}\right)
$$

Continuing this process, we finally obtain a connected plane curve $\widetilde{\Gamma}_{m}$ with the endpoints $\tilde{q}_{m}^{1}$ and $\tilde{q}_{m}^{2}$ satisfying that

$$
\begin{aligned}
\operatorname{dist}\left(p, \widetilde{\Gamma}_{m}\right) & >0, \\
\operatorname{dist}\left(p, \tilde{q}_{m}^{1}\right) & =\operatorname{dist}\left(p, \tilde{q}_{m}^{2}\right), \\
\operatorname{Angle}\left(\widetilde{\Gamma}_{m}, p\right) & =\operatorname{Angle}(\partial \Sigma, p), \\
\operatorname{Length}\left(\widetilde{\Gamma}_{m}\right) & =\operatorname{Length}(\partial \Sigma), \\
\operatorname{Area}\left(p \circledast \widetilde{\Gamma}_{m}\right) & =\operatorname{Area}(p \circledast \partial \Sigma) .
\end{aligned}
$$

Moreover, since $p \in \Sigma \backslash \partial \Sigma$, it follows from Proposition 3.1 that

$$
1 \leq \Theta(\Sigma, p)<\Theta(p \circledast \partial \Sigma, p) .
$$


Hence

$$
\begin{aligned}
\operatorname{Angle}\left(\widetilde{\Gamma}_{m}, p\right) & =\operatorname{Angle}(\partial \Sigma, p) \\
& =2 \pi \Theta(p \circledast \partial \Sigma, p) \\
& >2 \pi
\end{aligned}
$$

Therefore we get the conclusion from Lemma 3.3.

\section{Isoperimetric inequalities for soap-film-like surfaces in the hemisphere}

Bernstein [3] proved that a domain $\Sigma$ on the unit sphere $\mathbf{S}^{2}$ satisfies the sharp isoperimetric inequality

$$
4 \pi \operatorname{Area}(\Sigma)-\operatorname{Area}(\Sigma)^{2} \leq \operatorname{Length}(\partial \Sigma)^{2}
$$

One may guess that it would still hold for domains on any minimal surfaces in $\mathbf{S}^{n}$ as in $\mathbf{R}^{n}$. The answer to this problem is not yet known. However, for a point $p \in \mathbf{S}^{n}$, Choe and Gulliver [9] introduced the concept of a modified area $M_{p}(\Sigma)$ of a minimal surface $\Sigma$ in $\mathbf{S}^{n}$ and obtained an isoperimetric type inequality

$$
4 \pi M_{p}(\Sigma) \leq \operatorname{Length}(\partial \Sigma)^{2}
$$

where equality holds if and only if $\Sigma$ is a totally geodesic disk with center at $p$. In this section, we extend their results to soap-film-like surfaces in the upper hemisphere $\mathbf{S}_{+}^{n}:=\left\{x \in \mathbf{R}^{n+1}:|x|=1, x_{n+1}>0\right\}$.

Definition 4.1 [9]. Given a surface $\Sigma$ in $\mathbf{S}_{+}^{n}$, the modified area $M_{p}(\Sigma)$ of $\Sigma$ with center at a point $p \in \mathbf{S}_{+}^{n}$ is defined to be

$$
M_{p}(\Sigma)=\int_{\Sigma} \cos r .
$$

Remark 4.2. It is easy to see that $M_{p}(\Sigma) \leq \operatorname{Area}(\Sigma)$ for $\Sigma \subset \mathbf{S}_{+}^{n}$. For $p$, the north pole $(0, \ldots, 0,1)$, note that since $\cos r$ is the Jacobian of the projection of $\mathbf{S}_{+}^{n}$ into $x_{n+1}=0$, the modified area $M_{p}(\Sigma)$ is the Euclidean area of the orthogonal projection of $\Sigma$ into the horizontal hyperplane $x_{n+1}=0$, counting orientation [9].

Lemma 4.3 [9]. Let $\Sigma$ be a minimal surface in $\mathbf{S}_{+}^{n}$. Then for a fixed point $p \in \mathbf{S}_{+}^{n}$ the distance function $r(x)=\operatorname{dist}(p, x)$ in $\mathbf{S}_{+}^{n}$ satisfies the following. 
(a) $\Delta \cos r=-2 \cos r$,

(b) $2 \pi \delta_{p} \leq \Delta \log \frac{\sin r}{1+\cos r}$.

Proposition 4.4. For a strongly stationary surface $\Sigma \in \mathcal{S}$ in $\mathbf{S}_{+}^{n}$ and a point $p \in \mathbf{S}_{+}^{n}$, we have

$$
M_{p}(\Sigma) \leq M_{p}(p \circledast \partial \Sigma)
$$

Proof. As before, we use a classical minimal surface $\widetilde{\Sigma}$ obtained from $\Sigma$, such that $\partial \widetilde{\Sigma}=\partial \Sigma \cup\left(\cup C_{i}\right)$, where each $C_{i}$ is a piecewise $C^{1}$-curve. For a fixed $p \mathbf{S}_{+}^{n}$, the distance function $r(x)=\operatorname{dist}(p, x)$ satisfies

$$
\Delta \cos r=-2 \cos r
$$

by Lemma 4.3. Therefore

$$
\begin{aligned}
M_{p}(\Sigma)=M_{p}(\widetilde{\Sigma}) & =-\frac{1}{2} \int_{\widetilde{\Sigma}} \Delta \cos r \\
& =\frac{1}{2} \int_{\partial \widetilde{\Sigma}} \sin r \frac{\partial r}{\partial \nu} \\
& =\frac{1}{2} \int_{\partial \Sigma} \sin r \frac{\partial r}{\partial \nu} \\
& \leq \frac{1}{2} \int_{\partial \Sigma} \sin r \frac{\partial r}{\partial \eta} \\
& =-\frac{1}{2} \int_{p \circledast \partial \Sigma} \Delta \cos r \\
& =M_{p}(p \circledast \partial \Sigma) .
\end{aligned}
$$

As in Euclidean space, we have a density comparison inequality for a soap-film-like surface $\Sigma$ in $\mathbf{S}_{+}^{n}$ with nonclosed boundary as follows.

Proposition 4.5. Let $\Sigma \in \mathcal{S}$ be a strongly stationary surface in $\mathbf{S}_{+}^{n}$. Then for a point $p \in \mathbf{S}_{+}^{n}$, we have

$$
\Theta(\Sigma, p) \leq \Theta(p \circledast \partial \Sigma, p) .
$$


Proof. Since the distance function $r(x)=\operatorname{dist}(p, x)$ in $\mathbf{S}_{+}^{n}$ for a fixed point $p \in \mathbf{S}_{+}^{n}$ satisfies

$$
2 \pi \delta_{p} \leq \Delta \log \frac{\sin r}{1+\cos r}
$$

by Lemma 4.3, integrating the inequality (4.1) and using the divergence theorem, we have

$$
\begin{aligned}
2 \pi \Theta(\widetilde{\Sigma}, p) \leq \int_{\widetilde{\Sigma}} \Delta \log \frac{\sin r}{1+\cos r} & =\int_{\partial \widetilde{\Sigma}} \frac{1}{\sin r} \frac{\partial r}{\partial \nu} \\
& =\int_{\partial \Sigma} \frac{1}{\sin r} \frac{\partial r}{\partial \nu} \\
& \leq \int_{\partial \Sigma} \frac{1}{\sin r} \frac{\partial r}{\partial \eta} \\
& =\int_{p} \Delta \log \frac{\sin r}{1+\cos r} \\
& =2 \pi \Theta(p \nVdash \Sigma, p) .
\end{aligned}
$$

Theorem 4.6. Let $\Sigma \in \mathcal{S}$ be a strongly stationary surface in $\mathbf{S}_{+}^{n}$ with boundary $\partial \Sigma$. Assume that at least one component of $\partial \Sigma$ is a nonclosed curve with endpoints $a$ and $b$. Assume also that there exists a point $p \in \Sigma \backslash \partial \Sigma$, such that $\operatorname{dist}(p, a)=\operatorname{dist}(p, b)$ and $\partial \Sigma$ is radially connected from $p$. Then, we have the following.

$$
4 \pi M_{p}(\Sigma) \leq \operatorname{Length}(\partial \Sigma)^{2}
$$

Proof. As in the proof of Theorem 3.5, we can develop the cone $p \star \partial \Sigma$ on a two-dimensional great sphere $\mathbf{S}^{2} \subset \mathbf{S}^{n}$ and find a curve $\Gamma$ (not necessarily closed) in $\mathbf{S}^{2}$, such that $p \circledast \partial \Sigma$ is locally isometric to $\bar{p} \circledast \Gamma$ for $\bar{p}$ the north pole of $\mathbf{S}^{2}$ and the endpoint $q_{1}, q_{2}$ of $\Gamma$ satisfying that

$$
\operatorname{dist}\left(p, q_{1}\right)=\operatorname{dist}\left(p, q_{2}\right)
$$

Then

$$
\begin{aligned}
M_{p}(p \circledast \partial \Sigma) & =M_{\bar{p}}(\bar{p} \aleph \Gamma), \\
\operatorname{Length}(\partial \Sigma) & =\operatorname{Length}(\Gamma), \\
\operatorname{Angle}(\partial \Sigma, p) & =\operatorname{Angle}(\Gamma, \bar{p}) .
\end{aligned}
$$


As mentioned in Remark 4.2, $M_{\bar{p}}(\bar{p} \circledast \Gamma)$ is the same as the Euclidean area of the orthogonal projection of $\bar{p} \circledast \Gamma$ onto the two-plane containing the equator of $\mathbf{S}^{2}$. Let $\bar{\Gamma}$ be the image of $\Gamma$ under the projection and 0 the origin of the 2-plane. Then

$$
\begin{aligned}
M_{\bar{p}}(\bar{p} \circledast \Gamma) & =\operatorname{Area}(0 \circledast \bar{\Gamma}), \\
\operatorname{Angle}(\Gamma, \bar{p}) & =\operatorname{Angle}(\bar{\Gamma}, 0) .
\end{aligned}
$$

Moreover, since the projection is a length-decreasing map,

$$
\operatorname{Length}(\Gamma) \geq \operatorname{Length}(\bar{\Gamma}) .
$$

Note that if $\bar{q}_{1}$ and $\bar{q}_{2}$ are the endpoints of $\bar{\Gamma}$, then

$$
\operatorname{dist}\left(0, \bar{q}_{1}\right)=\operatorname{dist}\left(0, \bar{q}_{2}\right) .
$$

Applying Proposition 4.5, we see that

$$
\begin{aligned}
\operatorname{Angle}(\partial \Sigma, p) & =2 \pi \Theta(p \circledast \partial \Sigma, p) \\
& \geq 2 \pi \Theta(\Sigma, p) \\
& \geq 2 \pi .
\end{aligned}
$$

From Lemma 3.3 and Proposition 4.4, it follows that

$$
\begin{aligned}
4 \pi M_{p}(\Sigma) & \leq 4 \pi M_{p}(p \circledast \partial \Sigma) \\
& =4 \pi \operatorname{Area}(0 \circledast \bar{\Gamma}) \\
& \leq \operatorname{Length}(\bar{\Gamma})^{2} \\
& \leq \operatorname{Length}(\partial \Sigma)^{2},
\end{aligned}
$$

which completes the proof.

As a consequence of this theorem, for a soap-film-like surface with one boundary component, we have the following.

Corollary 4.7. Let $\Sigma \in \mathcal{S}$ be a strongly stationary surface in $\mathbf{S}_{+}^{n}$ with an embedded, connected, and nonclosed boundary curve $\Gamma$. Then for a point $p \in \Sigma \backslash \Gamma$, we have

$$
4 \pi M_{p}(\Sigma) \leq \text { Length }(\Gamma)^{2} .
$$




\section{Isoperimetric inequalities for soap-film-like surfaces in nonpositively curved spaces}

In 1999, Choe [8] proved that a minimal surface $\Sigma$ with one or two boundary components in a complete simply connected Riemannian manifold with sectional curvature bounded above by a nonpositive constant $K$ satisfies the sharp isoperimetric inequality

$$
4 \pi \operatorname{Area}(\Sigma)-K \text { Area }(\Sigma)^{2} \leq \operatorname{Length}(\partial \Sigma)^{2},
$$

where equality holds if and only if the minimal surface $\Sigma$ is a geodesic disk in a surface of constant Gaussian curvature $K$. In this section, we obtain an isoperimetric inequality for soap-film-like surfaces in a Riemannian manifold $M$ of sectional curvature bounded above by a constant $K$, applying Choe's method [8].

The key ingredient in the extension to the variable curvature case is to construct a suitable cone $\bar{p} \circledast \bar{\Gamma}$ associated with $\Sigma$ in a simply connected space form $\bar{M}$ of constant sectional curvature $K$. Thereafter, we derive the similar area and density estimates as in $\mathbf{R}^{n}$.

Lemma 5.1 [8]. Let $\Sigma$ be a minimal surface in a simply connected Riemannian manifold $M$ of sectional curvature bounded above by a constant $K$. Define $r(x)=\operatorname{dist}(p, x)$ for a fixed $p \in M$.

(I) If $K=0$, then we have on $\Sigma$ :

(a) $\Delta \log r \geq 2 \pi \delta_{p}$ if $p \in \Sigma$;

(b) $\Delta r \geq \frac{1}{r}$;

(II) If $K=-k^{2}<0$, then we have on $\Sigma$ :

(c) $\Delta r \geq k \operatorname{coth} k r$;

(d) $\Delta \log (1+\cosh k r) \geq-K$;

(e) $\Delta \log \frac{\sinh k r}{1+\cosh k r} \geq 2 \pi \delta_{p}$ if $p \in \Sigma$;

(f) $\Delta \log \sinh k r \geq 2 \pi \delta_{p}-K$ if $p \in \Sigma$;

(III) If $K=k^{2}>0$, then we have on $\Sigma$ :

(g) $\Delta \log \sin k r \geq 2 \pi \delta_{p}-K$ if $p \in \Sigma$ and $r \leq \frac{\pi}{2 k}$; 
(h) $\Delta r \geq k \cot k r$.

Theorem 5.2. Let $\Sigma \in \mathcal{S}$ be a strongly stationary surface with boundary $\partial \Sigma$ in a complete simply connected Riemannian manifold $M$ of sectional curvature bounded above by a nonpositive constant $K$. Assume that at least one component of $\partial \Sigma$ is a nonclosed curve with endpoints a and $b$. Assume also that there exists a point $p \in \Sigma \backslash \partial \Sigma$, such that $\operatorname{dist}(p, a)=\operatorname{dist}(p, b)$ and $\partial \Sigma$ is radially connected from $p$. Then we have

$$
4 \pi \operatorname{Area}(\Sigma)-K \operatorname{Area}(\Sigma)^{2} \leq \operatorname{Length}(\partial \Sigma)^{2}
$$

Proof. First, we assume $K=-k^{2}<0$. Define $r(x)=\operatorname{dist}(p, x), p \in \Sigma$. As before, we shall obtain some estimates for $\Sigma$ by using a classical minimal surface $\widetilde{\Sigma}$. Integrating Lemma 5.1.(d) over $\widetilde{\Sigma}$ gives

$$
\begin{aligned}
-K \operatorname{Area}(\Sigma) & =-K \operatorname{Area}(\widetilde{\Sigma}) \leq \int_{\widetilde{\Sigma}} \Delta \log (1+\cosh k r) \\
& =\int_{\partial \Sigma} \frac{k \sinh k r}{1+\cosh k r} \frac{\partial r}{\partial \nu}+\int_{\cup C_{i}} \frac{k \sinh k r}{1+\cosh k r} \frac{\partial r}{\partial \nu_{j}} \\
& \left.=\int_{\partial \Sigma} \frac{k \sinh k r}{1+\cosh k r} \frac{\partial r}{\partial \nu}, \quad \text { (by }(2.1)\right)
\end{aligned}
$$

where $\nu$ is the outward unit conormal to $\partial \Sigma$ and $\nu_{j}$ is the outward unit conormal to $\partial \Sigma_{j}$.

Let $\eta$ be the unit normal vector that makes the smallest angle with $\nabla r$. Then as in the inequality (3.5) it follows that

$$
\frac{\partial r}{\partial \nu} \leq \frac{\partial r}{\partial \eta}=\sqrt{1-\langle\nabla r, \tau\rangle^{2}}
$$

where $\tau$ is a unit tangent to $\partial \Sigma$ and thus we get

$$
-K \operatorname{Area}(\Sigma) \leq \int_{\partial \Sigma} \frac{k \sinh k r}{1+\cosh k r} \sqrt{1-\langle\nabla r, \tau\rangle^{2}} .
$$

We now construct the corresponding two-dimensional cone in the simply connected space form $\bar{M}$ of constant sectional curvature $K$ as follows. Let $\Gamma_{1}, \ldots, \Gamma_{m}$ be the components of $\partial \Sigma$, such that each $\Gamma_{i}$ is parameterized by $\Gamma_{i}(s)$ with arclength parameter $s$. Fix a point $\bar{p} \in \bar{M}$ and define $\bar{r}(y)=$ $\operatorname{dist}(\bar{p}, y), y \in \bar{M}$. Choose $q_{i} \in \Gamma_{i}$, for each $i=1, \ldots, m$. Then choose $\bar{q}_{i} \in$ $\bar{M}$ satisfying that $r\left(q_{i}\right)=\bar{r}\left(\bar{q}_{i}\right)$ for each $i$. We can find a curve $\bar{\Gamma}_{i} \subset \bar{M}$ 
parameterized by $\bar{\Gamma}_{i}(s)$ with arclength parameter $s \in\left[0, \operatorname{Length}\left(\Gamma_{i}\right)\right]$, such that

$$
\begin{aligned}
\bar{\Gamma}_{i}(0) & =\bar{q}_{i}, \\
r\left(\Gamma_{i}(s)\right) & =\bar{r}\left(\bar{\Gamma}_{i}(s)\right), \\
\left\langle r, \Gamma_{i}^{\prime}(s)\right\rangle & =\left\langle\bar{r}, \bar{\Gamma}_{i}^{\prime}(s)\right\rangle,
\end{aligned}
$$

where $\Gamma_{i}^{\prime}(s)$ and $\bar{\Gamma}_{i}^{\prime}(s)$ are the unit tangent vectors of $\Gamma_{i}(s)$ and $\bar{\Gamma}_{i}(s)$, respectively. It also follows from the definition of $\bar{\Gamma}_{i}$ that

$$
\operatorname{Length}\left(\Gamma_{i}\right)=\operatorname{Length}\left(\bar{\Gamma}_{i}\right)
$$

Hence

$$
\begin{aligned}
-K \operatorname{Area}(\Sigma) & \leq \sum_{i=1}^{m} \int_{\Gamma_{i}} \frac{k \sinh k r}{1+\cosh k r} \sqrt{1-\left\langle\nabla r, \Gamma_{i}^{\prime}(s)\right\rangle^{2}} \\
& =\sum_{i=1}^{m} \int_{\bar{\Gamma}_{i}} \frac{k \sinh k \bar{r}}{1+\cosh k \bar{r}} \sqrt{1-\left\langle\nabla \bar{r}, \bar{\Gamma}_{i}^{\prime}(s)\right\rangle^{2}} .
\end{aligned}
$$

Let $\bar{\eta}$ be the outward unit conormal to $\bar{\Gamma}_{i}$ on $\bar{p} \circledast \bar{\Gamma}_{i}$. Then it follows that

$$
\sqrt{1-\left\langle\nabla \bar{r}, \bar{\Gamma}_{i}^{\prime}(s)\right\rangle^{2}}=\frac{\partial \bar{r}}{\partial \bar{\eta}} .
$$

Furthermore, if $\bar{\Gamma}_{i}$ is not a closed curve in $\bar{M}$, the boundary of $\bar{p} \circledast \bar{\Gamma}_{i}$ consists of $\bar{\Gamma}_{i}$ and the two geodesic rays from $\bar{p}$ to $\bar{\Gamma}_{i}(0)$ and $\bar{\Gamma}_{i}\left(L_{i}\right)$, where $L_{i}=$ Length $\left(\Gamma_{i}\right)=\operatorname{Length}\left(\bar{\Gamma}_{i}\right)$. It is easy to see that $\frac{\partial \bar{r}}{\partial \bar{\eta}}=0$ on the geodesic rays. Therefore, we get

$$
\begin{aligned}
\operatorname{Area}(\Sigma) & \leq-\frac{1}{K} \sum_{i=1}^{m} \int_{\bar{\Gamma}_{i}} \frac{k \sinh k \bar{r}}{1+\cosh k \bar{r}} \frac{\partial \bar{r}}{\partial \bar{\eta}} \\
& =-\sum_{i=1}^{m} \int_{\bar{p} \circledast \bar{\Gamma}_{i}} \frac{1}{K} \Delta \log (1+\cosh k \bar{r}) \\
& =\sum_{i=1}^{m} \operatorname{Area}\left(\bar{p} \circledast \bar{\Gamma}_{i}\right) \\
& =\operatorname{Area}(\bar{p} \circledast \bar{\Gamma}), \bar{\Gamma}=\bigcup_{i=1}^{m} \bar{\Gamma}_{i}
\end{aligned}
$$


On the other hand, integrating Lemma 5.1.(e) over $\Sigma$ gives

$$
\begin{aligned}
2 \pi & \leq \int_{\widetilde{\Sigma}} \Delta \log \frac{\sinh k r}{1+\cosh k r}=\int_{\partial \widetilde{\Sigma}} \frac{k}{\sinh k r} \frac{\partial r}{\partial \nu} \\
& =\int_{\partial \Sigma} \frac{k}{\sinh k r} \frac{\partial r}{\partial \nu}+\int_{\cup C_{i}} \frac{k}{\sinh k r} \frac{\partial r}{\partial \nu} \leq \int_{\partial \Sigma} \frac{k}{\sinh k r} \frac{\partial r}{\partial \eta} \\
& =\int_{\bar{\Gamma}} \frac{k}{\sinh k \bar{r}} \frac{\partial \bar{r}}{\partial \bar{\eta}}=\int_{\bar{p} \circledast \bar{\Gamma}} \Delta \log \frac{\sinh k \bar{r}}{1+\cosh k \bar{r}}=\Theta(\bar{\Gamma}, \bar{p}) .
\end{aligned}
$$

Since $\bar{\Gamma}$ is also radially connected from $\bar{p}$ by our construction of $\bar{\Gamma}$, applying Lemma 3.3 gives

$$
4 \pi \operatorname{Area}(\bar{p} \circledast \bar{\Gamma}) \leq \operatorname{Length}(\bar{\Gamma})^{2}+K \operatorname{Area}(\bar{p} \circledast \bar{\Gamma})^{2} .
$$

Hence the desired isoperimetric inequality immediately follows from the inequality (5.2). Similarly we can prove the case of $K=0$.

Corollary 5.3. Let $M$ be a complete simply connected Riemannian manifold $M$ of sectional curvature bounded above by a nonpositive constant $K$. Let $\Sigma \in \mathcal{S}$ be a strongly stationary surface in $M$ with an embedded, connected, and nonclosed boundary curve $\Gamma$. Then we have

$$
4 \pi \operatorname{Area}(\Sigma)-K \operatorname{Area}(\Sigma)^{2} \leq \operatorname{Length}(\Gamma)^{2} .
$$

\section{Weak isoperimetric inequality for soap-film-like surfaces}

In [4, p. 318-319], Simon obtained a weaker isoperimetric inequality without connectedness assumption on boundary

$$
2 \pi \operatorname{Area}(\Sigma) \leq \text { Length }(\partial \Sigma)^{2}
$$

for a minimal surface $\Sigma$ in $\mathbf{R}^{n}$. Choe [8] proved a weaker isoperimetric inequality for a minimal surface $\Sigma$ in a simply connected Riemannian manifold $M$ with sectional curvature bounded above by a constant $K$ as follows:

$$
2 \pi \operatorname{Area}(\Sigma) \leq \operatorname{Length}(\partial \Sigma)^{2}+K \text { Area }(\Sigma)^{2} .
$$

Motivated by this, we shall obtain isoperimetric inequalities that are weaker than those in the previous sections but hold for all soap-film-like surfaces, which is an extension of Choe's result. 
Theorem 6.1. Let $\Sigma \in \mathcal{S}$ be a strongly stationary surface in a complete simply connected Riemannian manifold $M$ with sectional curvature bounded above by a constant $K$. If $K \leq 0$, then

$$
2 \pi \operatorname{Area}(\Sigma) \leq \operatorname{Length}(\partial \Sigma)^{2}+K \operatorname{Area}(\Sigma)^{2} .
$$

If $K>0$, the inequality (6.1) holds under the additional assumption that $\operatorname{diam}(\Sigma) \leq \frac{\pi}{2 \sqrt{K}}$

Proof. We first prove the inequality (6.1) when $K=-k^{2}<0$. As before, we consider a minimal surface $\widetilde{\Sigma}$ in $M$ with $\partial \widetilde{\Sigma}=\partial \Sigma \cup\left(\cup C_{i}\right)$, where $\cup C_{i}$ is the set of the singular curves. Integrating Lemma 5.1.(e) over $\widetilde{\Sigma}$, we get

(6.2) $2 \pi-K \operatorname{Area}(\widetilde{\Sigma}) \leq \int_{\widetilde{\Sigma}} \Delta \log \sinh k r=\int_{\partial \widetilde{\Sigma}} k \operatorname{coth} k r \leq \int_{\partial \Sigma} k \operatorname{coth} k r$.

Moreover using Lemma 5.1.(b), integrating the inequality (6.2) over $\widetilde{\Sigma}$ and applying Fubini's theorem, we obtain

$$
\begin{aligned}
2 \pi \operatorname{Area}(\widetilde{\Sigma})-K \operatorname{Area}(\widetilde{\Sigma})^{2} & \leq \int_{\widetilde{\Sigma}} \int_{\partial \Sigma} k \operatorname{coth} k r \\
& =\int_{\partial \Sigma} \int_{\widetilde{\Sigma}} k \operatorname{coth} k r \\
& \leq \int_{\partial \Sigma} \int_{\widetilde{\Sigma}} \Delta r \leq \int_{\partial \Sigma} \int_{\partial \Sigma} \frac{\partial r}{\partial \nu}=\operatorname{Length}(\partial \Sigma)^{2} .
\end{aligned}
$$

When $K=0$, we obtain the inequality (6.1) by integrating Lemma 5.1.(a) twice and applying Lemma 5.1.(b).

When $K>0$, we obtain the inequality (6.1) by integrating Lemma 5.1.(g) twice and applying Lemma 5.1.(h).

For a strongly stationary surface $\Sigma \in \mathcal{S}$ in $\mathbf{R}^{n}$, the above Theorem 6.1 implies that

$$
2 \pi \operatorname{Area}(\Sigma) \leq \operatorname{Length}(\Gamma)^{2}
$$

However, using Stone's idea [19], we can improve the inequality (6.3) by the constant $\sqrt{2}$ as follows.

Theorem 6.2. Let $\Sigma \in \mathcal{S}$ be a strongly stationary surface in $\mathbf{R}^{n}$. Then we have

$$
2 \sqrt{2} \pi \operatorname{Area}(\Sigma) \leq \operatorname{Length}(\partial \Sigma)^{2}
$$


Before we prove this theorem, we need the following flux formula for soap-film-like surfaces.

Lemma 6.3 Flux formula. Let $\Sigma \in \mathcal{S}$ be a strongly stationary surface in $\mathbf{R}^{n}$. Then we have

$$
\int_{\partial \Sigma} \nu=0
$$

where $\nu$ is the outward unit vector conormal to $\partial \Sigma$.

Proof. Let $\cup C_{i}$ be the set of the singular curves in $\Sigma$. For a minimal surface $\widetilde{\Sigma}$ which is obtained from $\Sigma$ by cutting $\Sigma$ along the singular curves $\cup C_{i}$, the well-known flux formula says that

$$
\int_{\partial \widetilde{\Sigma}} \nu=0
$$

Since $\partial \widetilde{\Sigma}=\partial \Sigma \cup\left(\cup C_{i}\right)$, we get

$$
0=\int_{\partial \widetilde{\Sigma}} \nu=\int_{\partial \Sigma} \nu+\int_{\cup C_{i}} \nu_{j}=\int_{\partial \Sigma} \nu
$$

where $\nu_{j}$ is the outward unit conormal to $\partial \Sigma_{j}$ along $\cup C_{i}$ where the surfaces $\Sigma_{j}$ 's meet.

Proof of Theorem 6.2. Let $C=\cup C_{i}$ be the set of the singular curves in $\Sigma$. We cut $\Sigma$ along the singular curves $\cup C_{i}$ on $\Sigma$ to get $\widetilde{\Sigma}$ satisfying that $\widetilde{\Sigma}$ is a minimal surface in $\mathbf{R}^{n}$ with $\partial \tilde{\Sigma}=\partial \Sigma \cup\left(\cup C_{i}\right)$. Integrating Lemma 5.1.(a) over $\widetilde{\Sigma}$ gives

$$
2 \pi \leq \int_{y \in \widetilde{\Sigma}} \Delta \log r_{x}(y) \leq \int_{y \in \partial \widetilde{\Sigma}} \frac{1}{r_{x}(y)} \frac{\partial r_{x}(y)}{\partial \nu} \leq \int_{y \in \partial \Sigma} \frac{1}{r_{x}(y)}
$$

$\underset{\sim}{\text { where }} r_{x}(y)=\operatorname{dist}(x, y)$ for a fixed $x \in \Sigma$. Integrating Lemma 5.1.(b) over $\widetilde{\Sigma}$ gives

$$
\int_{x \in \widetilde{\Sigma}} \frac{1}{r_{y}(x)} \leq \int_{x \in \widetilde{\Sigma}} \Delta r_{y}(x) \leq \int_{x \in \partial \widetilde{\Sigma}} \frac{\partial r_{y}(x)}{\partial \nu}=\int_{x \in \partial \Sigma} \frac{\partial r_{y}(x)}{\partial \nu}
$$


Integrating the inequality (6.4) over $\widetilde{\Sigma}$ and using Fubini's theorem and the inequality (6.5) we obtain

$$
\begin{aligned}
2 \pi \operatorname{Area}(\Sigma) \leq \int_{x \in \widetilde{\Sigma}} \int_{y \in \partial \Sigma} \frac{1}{r_{x}(y)} & =\int_{y \in \partial \Sigma} \int_{x \in \widetilde{\Sigma}} \frac{1}{r_{x}(y)} \\
& =\int_{y \in \partial \Sigma} \int_{x \in \widetilde{\Sigma}} \frac{1}{r_{y}(x)} \\
& \leq \int_{y \in \partial \Sigma} \int_{x \in \partial \widetilde{\Sigma}} \frac{\partial r_{y}(x)}{\partial \nu} \\
& =\int_{y \in \partial \Sigma} \int_{x \in \partial \Sigma} \frac{\partial r_{y}(x)}{\partial \nu}
\end{aligned}
$$

Since the roles of $x$ and $y$ can be interchanged in the inequality (6.6), we also have

$$
2 \pi \operatorname{Area}(\Sigma) \leq \int_{x \in \partial \Sigma} \int_{y \in \partial \Sigma} \frac{\partial r_{x}(y)}{\partial \nu} .
$$

Adding up the inequalities (6.6) and (6.7) we get

$$
4 \pi \operatorname{Area}(\Sigma) \leq \int_{y \in \partial \Sigma} \int_{x \in \partial \Sigma} \frac{\partial r_{y}(x)}{\partial \nu}+\frac{\partial r_{x}(y)}{\partial \nu} .
$$

Note that

$$
\frac{\partial r_{y}(x)}{\partial \nu}=\left\langle\nabla r_{y}(x), \nu(x)\right\rangle=\left\langle\frac{x-y}{|x-y|}, \nu(x)\right\rangle .
$$

Hence, the inequality (6.8) becomes

$$
\begin{aligned}
4 \pi \operatorname{Area}(\Sigma) \leq & \int_{y \in \partial \Sigma} \int_{x \in \partial \Sigma}\left\langle\frac{x-y}{|x-y|}, \nu(x)\right\rangle+\left\langle\frac{x-y}{|x-y|},-\nu(y)\right\rangle \\
\leq & \int_{y \in \partial \Sigma} \int_{x \in \partial \Sigma}|\nu(x)-\nu(y)| \\
\leq & \operatorname{Length}(\partial \Sigma)\left(\int_{y \in \partial \Sigma} \int_{x \in \partial \Sigma}|\nu(x)-\nu(y)|^{2}\right)^{\frac{1}{2}} \\
& \quad \text { by the Hölder inequality) } \\
= & \text { Length }(\partial \Sigma)\left(\int_{y \in \partial \Sigma} \int_{x \in \partial \Sigma}(2-2\langle\nu(x), \nu(y)\rangle)\right)^{\frac{1}{2}} \\
= & \operatorname{Length}(\partial \Sigma)\left(\int_{y \in \partial \Sigma} \int_{x \in \partial \Sigma} 2\right)^{\frac{1}{2}} \quad \text { (by Lemma 6.3) } \\
= & \sqrt{2} \operatorname{Length}(\partial \Sigma)^{2} .
\end{aligned}
$$




\section{Acknowledgments}

The author thanks the referee for his/her careful reading of the manuscript and for many helpful suggestions. The author also thanks Professor Jaigyoung Choe for helpful discussions and valuable comments. This research was supported by the Sookmyung Women's University Research Grants 2012 .

\section{References}

[1] F. Almgren, Optimal isoperimetric inequalities, Indiana Univ. Math. J. 35(3) (1986), 451-547.

[2] F. Almgren and J. Taylor, Geometry of soap films, Sci. Am. 235 (1976), 82-93.

[3] F. Bernstein, Über die isoperimetrische Eigenschaft des Kreises auf der Kugeloberfläche und in der Ebene, Math. Ann. 60(1) (1905), 117-136.

[4] E. Bombieri, An introduction to minimal currents and parametric variational problems, Math. Rep. 2(3) (1985), 285-384.

[5] T. Carleman, Zur Theorie der Minimalflächen, Math. Z. 9(1-2) (1921), 154-160.

[6] J. Choe, The isoperimetric inequality for a minimal surface with radially connected boundary, Ann. Scuola Norm. Sup. Pisa (4) 17(4) (1990), 583-593.

[7] J. Choe, Sharp isoperimetric inequalities for stationary varifolds and area minimizing flat chains mod $k$, Kodai Math. J. 19(2) (1996), $177-190$.

[8] J. Choe, The isoperimetric inequality for minimal surfaces in a Riemannian manifold, J. Reine Angew. Math. 506 (1999), 205-214.

[9] J. Choe and R. Gulliver, Isoperimetric inequalities on minimal submanifolds of space forms, Manuscripta Math. 77(2-3) (1992), 169189.

[10] J. Drachman and B. White, Soap films bounded by nonclosed curves, J. Geom. Anal. 8(2) (1998), 239-250. 
[11] T. Ekholm, B. White and D. Wienholtz, Embeddedness of minimal surfaces with total boundary curvature at most $4 \pi$, Ann. Math. 155(1) (2002), 209-234.

[12] J. Feinberg, The isoperimetric inequality for doubly-connected minimal surfaces in $\mathbf{R}^{n}$, J. Anal. Math. 32 (1977), 249-278.

[13] M. Gromov, Filling Riemannian manifolds, J. Differ. Geom. 18(1) (1983), 1-147.

[14] R. Gulliver and S. Yamada, Area density and regularity for soap film-like surfaces spanning graghs, Math. Z. 253(2) (2006), 315-331.

[15] P. Li, R. Schoen and S.-T. Yau, On the isoperimetric inequality for minimal surfaces, Ann. Scuola Norm. Sup. Pisa Cl. Sci. (4) $\mathbf{1 1}(2)$ (1984), 237-244.

[16] R. Osserman and M. Schiffer, Doubly connected minimal surfaces, Arch. Rational Mech. Anal. 58(4) (1975), 285-307.

[17] H. Parks, Soap-film-like minimal surfaces spanning knots, J. Geom. Anal. 2(3) (1992), 267-290.

[18] L. Simon, Lectures on geometric measure theory, Proc. Centre Math. Anal. Austr. Natl Univ. Vol. 3, Canberra, Australia, 1983.

[19] A. Stone, On the isoperimetric inequality on a minimal surface, Calc. Var. Partial Differ. Equ. 17(4) (2003), 369-391.

[20] J. Taylor, The structure of singularities in soap-bubble-like and soap-film-like minimal surfaces, Ann. Math. 103(3) (1976), 489-539.

KeOMKYo SEO

Department of Mathematics

SOOKMYUNG WOMEN's UNIVERSITY

HYOCHANGWONGIL 52

Yongsan-Ku, SeOul 140-742, KoreA

E-mail address: kseo@sookmyung.ac.kr

$U R L$ : http://sookmyung.ac.kr/ kseo

Received December 14, 2011 
